\title{
Implications of IPV Introduction in National Immunization Schedule and Strategies to Combat Shortages
}

\author{
Sharma Pragya*, Rathi Akanksha, Taneja DK, Garg Suneela \\ Department of Community Medicine, Maulana Azad Medical College, New Delhi, India
}

Received: April 30, 2016; Accepted: May 10, 2016; Published: June 28, 2016

*Corresponding author: Dr. Pragya Sharma, Associate Professor, Dept. of Community Medicine, Maulana Azad Medical College, New Delhi, India, E-mail: drpragyaS@gmail.com

\begin{abstract}
With the upcoming globally synchronized switch from $\mathrm{tOPV}$ to $\mathrm{b}$ OPV for commitment of countries to be free from $t$ OPV, 156 countries have pledged to stop using the same and replace it either with $\mathrm{b}$ OPV and IPV combination or only IPV. Thus there is humongous demand of IPV against the production capacity of the vaccine across the globe. There is an urgent need to explore various strategies to combat the forthcoming crisis in the given demand supply shortage and also in the post eradication era. Also it is important to consider the cost effective strategies for the middle and low income countries for sustainability once IPV has been included in the programme.
\end{abstract}

\section{Introduction}

Vaccination has greatly reduced the burden of infectious diseases. Only clean water, also considered to be a basic human right, performs better (1). The strongest evidence of the effectiveness of vaccination is eradication of Smallpox and neareradication of Polio. The world is in the last leg in its fight against Polio and the most important tool behind the success of this fight has been the Oral Poliovirus Vaccine (OPV). However, there have been some perils of the widespread use of OPV due to its live attenuated nature - namely Vaccine Associated Paralytic Polio (VAPP) and Vaccine Derived Polio Virus (VDPV). Out of the three strains of polio virus $(1,2 \& 3)$, strain 2 has been long eradicated and no case of wild polio virus 2 has been reported globally since 1999. However, thousands of children got afflicted with VAPP and around 600 with type $2 \mathrm{cVDPV}$ because of the continued use of trivalent OPV since 1999 (2). This mandated a strategy for sequential withdrawal of OPV vaccine and at the same time ensure high immunity of the population. Polio Eradication and End Game Strategic Plan 2013-2018 has been developed by the Global Polio Eradication Initiative (GPEI) in consultation with national health authorities, global health initiatives, scientific experts, donors and other stakeholders, in response to a directive of the World Health Assembly (3). This plan deals with the eradication and containment of polio caused not just by wild viruses but also cases associated with oral polio vaccine (OPV) (4). Since $40 \%$
VAPP and 97\% VDPV is caused by strain 2 (6). so the first major step being taken is the 'SWITCH' from trivalent Oral Poliovirus Vaccine ( $\mathrm{t}$ OPV) to bivalent Oral Poliovirus Vaccine (b OPV) at the global level. This switch will solve the problem of VAPP \& VDPV to a great extent but at the same time the immunity of the subsequent birth cohorts against strain 2 will plummet due to the absence of the same from the vaccine and there is a risk of outbreaks of cVDPV type 2 as it is circulating in the population due to use of $\mathrm{t} \mathrm{OPV}$ in the national programme. The strategy to mitigate this threat is simultaneous introduction of one dose of Injectable Poliovirus Vaccine (IPV) at 14 weeks along with $3^{\text {rd }}$ dose of Diptheria, Pertussis and Tetanus (DPT3) vaccine that has a dual advantage due to its killed nature - first is that it does not have potential to cause VAPP or VDPV and the other is that it maintains the immunity level of the individual against all the three strains of the virus (since it has all 3 strains).

With the upcoming globally synchronized switch over a period of two weeks, from $t$ OPV to b OPV for commitment of countries to be free from $t$ OPV, 156 countries have pledged to stop using the same and replaced it either with b OPV and IPV combination or only IPV. India falls in the former category and has observed the switch on $25^{\text {th }}$ April 2016 leading to a complete cessation of administration of $t$ OPV to eligible children by government hospitals, dispensaries, health centers, healthcare workers and private practitioners. The date of $9^{\text {th }}$ May 2016 will be observed as the national validation day and all vaccine storage points - be it Primary Health Centers (PHCs), Community Health Centers (CHCs), Sub-district hospitals, District hospitals, Tertiary care hospitals or any other storage point would have to return all vials of $\mathrm{t}$ OPV to district headquarters for destruction.

As a risk mitigation strategy to prevent possible sabin type 2 Poliovirus outbreaks by building an immunity base to polio virus type 2 and strengthen immunity against type 1 and 3, phased introduction of single dose of IPV two weeks prior has been recommended. Sequential administration of IPV and b OPV boosts local as well as humoral immunity.The efficacy of 3 doses of IPV is almost $100 \%$ against all 3 strains as compared to a single dose that provides protection to $32 \%-63 \%$ of the recipients 
against strain 2.(6) However the decision for introducing just one dose of IPV as compared to the usual 3-dose schedule is not purely based on economics due to the humongous amount of doses required to immunize the whole birth cohorts of 150 plus countries but also has a scientific rationale.(6) Besides, there are various reasons for clubbing IPV with DPT3 like interference by maternal antibodies if given at an earlier age, priming of infants with OPV, better compliance by the vaccinee due to same number of visits, no additional burden on logistics and manpower.

\section{No. of Doses Required}

According to 2016 India factsheet of Global Alliance for Vaccine and Immunization (GAVI) (5), the total population of India is 1.32 billion and the birth cohort is 25.8 million. Considering the current Infant Mortality Rate of 40 (SRS 2013), the number of infants surviving annually are about 24.8 million. From the data for coverage for DPT3 (5), which is $88 \%$, and the predicted wastage factor for the IPV vaccine 1.33;

The estimated number of doses required $(\mathrm{N})=$ (population to be vaccinated) * (expected coverage) $*$ (number of IPV dose in the schedule) $*$ (wastage factor)

$$
\mathrm{N}=24,800,000 * 0.88 * 1 * 1.33=29,025,920
$$

Thus the number of doses of IPV required to vaccinate the whole birth cohort in year 2016 is nearly 29 million doses annually for India alone.

The number of doses required for the subsequent years will remain the same (if the birth rate/infant mortality rate is the same) as unlike other vaccines there is no catch up vaccination involved in IPV.

\section{Cost Implications of introducing a single dose of IPV}

In 2014, India applied for IPV support to GAVI for a period of 3 years from 2015 to 2018 for a total of US\$160M.(8) In 2015, GAVI approved the IPV support to India for a period of one year with recommendations that the country will have to self-finance the program after the one-year period is over. India had asked for 5 -dose vials but due to shortage of the same, however currently, a combination of 5-dose and 10-dose vials has been provided for use.

The government of India is acquiring the 10-dose vials of IPV at a unit cost of US\$10. Thus one dose of IPV costs around US $\$ 1$ or approximately 62 INR. Thus amounting to a total direct cost implication for vaccinating the birth cohort as US\$29M or 1,798 million INR. Besides other direct and indirect capital costs attributed to infrastructure and equipment, human resource, procurement of $\mathrm{AD}$ syringes, cold chain equipments etc., administration, overhead costs and cost of treating side effects need to be calculated.(7)

The availability of IPV is already in short supply globally and then with such a huge requirement coming up owing to its inclusion in the Universal Immunization Programme (UIP) of the country, the issue of sustenance of vaccine into the programme after introduction has to be carefully looked into. In the given situation when vaccine demand is also globally synchronized, a further higher probability of vaccine shortage can be fore casted following initial introduction. If we look at the global demand of the IPV vaccine it amounts to approximately 80 million doses currently and can go up to 190-425 million doses in post eradication era. However, it is estimated that existing IPV production capacity is only about 120 million doses globally.

Unless the strategies to combat the forthcoming crisis are in place, this situation can play a reversal in the entire game of eradication of the disease.

\section{Strategies to combat shortages in IPV supply}

Promoting domestic players: Reducing taxes and increasing incentives for the vaccine manufacturing companies will encourage them to manufacture the needed vaccines. Government has an important role to play in the vaccine industry. Its work falls under three areas broadly - policy, regulatory and promotion of research through funding. (13) However, this is a time consuming process and vaccine is unlikely to be available in next two-three years.

Alternative route for vaccination: Intra-dermal delivery (IDD) will lead to reduced volumes of vaccine per dose (9). IDD of reduced doses $(20 \%$ of the standard volume and therefore $20 \%$ of the standard antigen content) induced "striking" antibody responses in adults and children who had previously been immunized (10). Two intra-dermal doses (each $20 \%$ of that required to be given through I/M route) of IPV given 4-8 weeks apart rendered higher protective immunity at $1 / 5^{\text {th }}$ of the full IPV dose (Revised WHO Position paper, publication date $25^{\text {th }}$ March). However, the downside of giving intra-dermal injections is the requirement of trained manpower or intra-dermal adapters that will further increase the cost.

Saving the antigen: Use of adjuvants to allow a reduced IPV antigen content per dose (9). The current formulation that is being provided in the immunization schedule doesn't contain any adjuvant. Adding adjuvants to the vaccine leads to decreased need of antigen and more vaccine can be produced from lesser amount of antigen. It has been estimated that incorporation of 'Aluminum-based adjuvants' might enable a three- to four-fold lower IPV content to be used.(11)

Reduce vaccination costs by using IPV in combination vaccines (9): Apart from the stand-alone IPV formulations; IPV is also available in the form of a Hexavalent vaccine having 6 antigens namely Diphtheria, Pertussis, Tetanus, Hemophilus Influenza, Polio and Hepatitis-B. Hexavalent combinations should result in savings due to administration of fewer injections, reduction in cost of syringes and their disposal, reduction in cold chain costs, and reductions in vaccine waste (12). The downside of using hexavalent vaccine is that the pertussis antigen used in this vaccine is a cellular rather than the whole-cell antigen which is more antigenic and thus recommended for primary immunization.

Development of heat-stable vaccine: on the lines of other vaccines like heat-stable OPV vaccine. It has two advantages - 
first it prevents the wastage of vaccine due to heat exposure and second it reduces the burden on the already burdened cold chain system.

Injectable Sabin Virus Vaccine(s IPV): IPV containing the attenuated strains of Sabin poliovirus (s IPV) is less expensive vaccine and has a likely lower manufacturing cost in countries where oral polio vaccine is already manufactured like China, India, and Indonesia as compared to Europe and United States. Besides this would also reduce the potential severity of the consequences of intentional or unintentional release of virus from an IPV manufacturing facility (9).

Introduction of freeze indicators: The IPV vaccine is not an adsorbed vaccine like DPT, DT, Hep-B or TT vaccine thus it won't sediment upon thawing after freezing. Hence, shake test doesn't apply for this vaccine. In case there is a doubt that that the vaccine has been frozen, it must be discarded as there is no way to confirm the same. These on vials will be to minimize wastage in case of ambiguity.

These strategies would be more pertinent even in the posteradication era as OPV would have to be completely withdrawn and only IPV schedule will be followed. In such a scenario, 3-4 doses of IPV will be required per child increasing the IPV demand to 87-116 million doses per year for India alone (9). As of February 2016, Gavi has assured 28.14 million doses of IPV (14). Domestic procurement from the Hyderabad-based Shantha Biotech for the period October 2016 to March 2018 will provide another 24 million doses, leaving a shortfall of 23.42 million doses, notes the IEAG (14) freeze indicators on vials will lead to minimising wastage in case of ambiguity. It will require few more years before the manufacturing companies can expand their vaccine producing capacity thus mandating adoption strategies that reduce the vaccine demand at the earliest.

\section{Current Domestic Production of IPV}

Indian pharmaceutical companies have a large market share in vaccines. They provide low-cost solutions to the vaccines included in national immunization schedule. The reason being that there is abundant skilled manpower, low cost research \& development, humongous demand and healthy competition However, out of the top 6 pharmaceutical companies (Serum Institute, Panacea Biotech, Bharat Biotech, Indian Immunologicals, Shantha Biotech \& Biological E), only 2 (Panacea Biotech \& Immunological E) are currently producing IPV vaccine and 1 (Shantha Biotech) is planning to produce the same in future (13).

\section{Conclusion}

The path to achieving self-sufficiency in IPV vaccination is long and hard. The support from GAVI will end soon and the various strategies to reduce the vaccine requirement have to be explored. The domestic pharmaceutical industry should stand up to increase production to meet the national requirements to produce a cheaper substitute of the already subsidized IPV vaccine, and that too in the given time frame.

\section{References}

1. Plotkin SL, Plotkin SA. A short history of vaccination. In: Plotkin SA, Orenstein WA, eds. Vaccines, 4th ed. Philadelphia: WB Saunders; 2004:1-15.

2. Jog P. Goodbye Switch and Imminent Polio Victory. President's page. Indian Pediatr [Internet]. 2016;53.

3. Global Polio Eradication Initiative $>$ Resource library $>$ Strategy and work [Internet]. Polioeradication.org. 2016

4. Polio Global Eradication Initiative. Introduction of Inactivated Polio

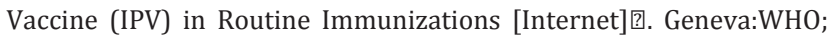
2014.

5. Gavi country factsheet : India [Internet]. Gavi.org. 2016.

6. Polio Global Eradication Initiative. Background and Technical Rationale for Introduction of One dose of Inactivated Polio Vaccine (IPV) in Routine Immunization Schedule [Internet]. Geneva:WHO; 2014.

7. Dhanasiri SK, Puliyal JM. Regulating vaccines: can health economics tools be used profitable? [letter]. Indian Pediatr. 2007; 44(1):11-14.

8. Berckley S. India and IPV -Board meeting. Geneva: GAVI; 10-11 Dec 2014

9. Improving the affordability of vaccines by low and middle income countries [Internet]. Seattle: PATH; 2010.

10. Samuel BU, Cherian T, Sridharan G, Mukundan P, John TJ. Immune response to intradermally injected inactivated poliovirus vaccine. Lancet. 1991 Aug 10;338(8763):343-344.

11. Sutter R. 8th WHO/UNICEF Consultation with OPV/IPV Manufacturers and National Regulatory Authorities. Presentation presented at 2009. Geneva.

12. Wyman O. Global Post-eradication IPV Supply and Demand Assessment: Integrated Findings. Seattle: Bill and Melinda Gates Foundation. 2009.

13. Sharma A. Vaccines market in India [Internet]. Delhi: Netherlands Office of Science \& Technology. 2013

14. Prasad R. Off-label use of IPV vaccine to tide over shortage. The Hindu [newspaper online]. 2016. 